\title{
AVALIAÇÃO DOS PROBLEMAS RELACIONADOS AO ALEITAMENTO MATERNO A PARTIR DO OLHAR DA ENFERMAGEM
}

\author{
Manoel Dias de Souza Filho1, Pedro Nolasco Tito Gonçalves Neto², Maria do Carmo de Carvalho e Martins ${ }^{3}$
}

RESUMO: Estudo qualitativo com inspiração fenomenológica, cujo objetivo foi investigar os problemas relacionados ao aleitamento, e realizado com dez membros da equipe de enfermagem de uma maternidade pública de Teresina, Estado do Piauí. As informações foram obtidas por meio de entrevista com a abordagem de conteúdos teóricos e habilidades para o cuidado e problemas relacionados ao aleitamento, e interpretados mediante análise temática. Emergiram como categorias: problemas relacionados ao aleitamento, medidas adotadas pela equipe de enfermagem para prevenção dos problemas maternos relacionados ao aleitamento, e responsabilidade da equipe de enfermagem na prevenção dos problemas maternos relacionados ao aleitamento. Os profissionais buscam, por meio de medidas educativas e de promoção de saúde, ajudar as mulheres a desenvolver estratégias para prevenção de problemas no aleitamento materno.

PALAVRAS-CHAVE: Educação em enfermagem; Aleitamento materno; Desmame.

\section{EVALUATION OF PROBLEMS RELATED TO BREASTFEEDING FROM THE NURSING PERSPECTIVE}

\begin{abstract}
A qualitative study from a phenomenological angle with the objective of investigating problems related to breastfeeding, it was carried out with ten members of the nursing team of a state maternity unit in Teresina, in the state of Piauí. Data was gathered through interviews, with an approach based around theoretical content and abilities for caring for nursing mothers and dealing with the problems they may have, and interpreted through thematic analysis. The following categories emerged: problems related to breastfeeding, measures adopted by the nursing team to prevent problems experienced by nursing mothers, and the responsibility of the nursing team for preventing those problems. Through education and health promotion, the health professionals seek to help women to develop strategies for preventing problems related to breastfeeding. KEYWORDS: Nursing education; Breastfeeding; Weaning.
\end{abstract}

\section{EVALUACIÓN DE LOS PROBLEMAS ACERCA DEL AMAMANTAMIENTO MATERNO A PARTIR DE LA ÓPTICA DE LA ENFERMERÍA}

RESUMEN: Estudio cualitativo con inspiración fenomenológica, cuyo objetivo fue investigar los problemas del amamantamiento. Fue realizado con diez miembros del equipo de enfermería de una maternidad pública de Teresina, estado de Piauí. Las informaciones fueron obtenidas por medio de entrevista con abordaje de contenidos teóricos y habilidades para el cuidado y problemas relacionados al amamantamiento, e interpretados por medio de análisis temático. Surgieron como categorías: Problemas acerca del amamantamiento, Medidas adoptadas por el equipo de enfermería para prevención de los problemas maternos referentes al amamantamiento, y Responsabilidad del equipo de enfermería en la prevención de los problemas maternos relacionados al amamantamiento. Los profesionales buscan, por medio de medidas educativas y de promoción de salud, ayudar a las mujeres a desarrollar estrategias para prevención de problemas en el amamantamiento materno. PALABRAS CLAVE: Educación en enfermería; Amamantamiento materno; Destete.

\footnotetext{
${ }^{1}$ Cirurgião-dentista. Mestre em Ciências e Saúde. Professor de Ciências Fisiológicas da Universidade Federal do Piauí-UFPI .

${ }^{2}$ Enfermeiro.

${ }^{3}$ Nutricionista. Doutora em Ciências Biológicas. Professora do Departamento de Biofísica e Fisiologia e do Programa de Pós-Graduação em Alimentos e Nutrição da UFPI. Professora da Faculdade de Saúde, Ciências Humanas e Tecnológicas do Piauí - NOVAFAPI.
}

\author{
Autor correspondente: \\ Manoel Dias de Souza Filho \\ Universidade Federal do Piauí \\ R. Equador, 118 - 64215-620 - Parnaíba-PI-Brasil \\ E-mail: manoeldias@ufpi.edu.br
}

Recebido: $23 / 07 / 10$ Aprovado: $10 / 02 / 11$ 


\section{INTRODUÇÃO}

As propriedades nutricionais do leite materno, o aspecto protetor contra infecções, bem como o fato de que o aleitamento favorece o vínculo afetivo entre mãe e filho são amplamente divulgados ${ }^{(1)}$. Em estudo sobre prevalência do aleitamento materno realizado nas capitais brasileiras e no Distrito Federal pelo Ministério da Saúde, evidenciou-se que a maioria das crianças é amamentada no primeiro mês de vida (87\%), porém, considerados os dias de vida, apenas $35 \%$ das crianças recebem o leite materno $^{(2)}$

Além dos fatores culturais, educativos e sociais relativas ao aleitamento materno, doenças envolvendo a mãe podem constituir obstáculos importantes à amamentação. A má técnica de amamentação, mamadas infrequentes e em horários pré-determinados, constituem importantes fatores que podem predispor o aparecimento de complicações da lactação, tais como: ingurgitamento mamário, traumas mamilares e baixa produção de leite, uma vez que constituem-se em condições que levam a um esvaziamento mamário inadequado ${ }^{(3)}$.

Nessas circunstâncias, é importante que o profissional de enfermagem tenha habilidade, conhecimento técnico e atitude acolhedora para avaliar, adequadamente, a viabilidade da amamentação ${ }^{(4)}$. Situações especiais e dificuldades na amamentação podem contribuir para que a mãe se sinta temerosa e desestimulada, desmamando seu filho mais precocemente $^{(5)}$.

Contudo, não basta que a mulher esteja informada das vantagens do aleitamento materno e faça opção por esta prática, ela precisa estar inserida em um ambiente favorável à amamentação e encontrar apoio no profissional de saúde. Considerando que o início da lactação ocorre, frequentemente, no hospital, é de responsabilidade dos profissionais de saúde proporcionarem às mães orientações e conhecimentos técnicos e demonstrar interesse à prática da amamentação, criando afeto entre mãefilho $^{(6)}$.

A realização do presente estudo justificou-se pela importância da equipe de enfermagem na promoção do aleitamento materno, sendo necessária a discussão sobre as demandas da assistência nesse processo. $\mathrm{O}$ estudo procurou identificar os principais problemas relacionados ao aleitamento materno, segundo a visão da equipe de enfermagem, frente às dificuldades das mães no ato da amamentação em um hospital público municipal da Cidade de Teresina, Estado do Piauí.

\section{METODOLOGIA}

Realizou-se um estudo fenomenológico pelo fato de que, por capturar a experiência vivida, a abordagem qualitativa é uma alternativa de investigação, em substituição aos métodos tradicionais utilizados pelas ciências naturais ${ }^{(7)}$. As abordagens qualitativas abarcam a totalidade dos seres humanos, concentrando-se na experiência huma$\mathrm{na}^{(8)}$. Ainda, permitem apresentar contribuições no processo de mudança, criação ou formação de opiniões de determinado grupo e, em maior grau de profundidade, interpretar particularidades dos comportamentos ou atitudes dos indivíduos ${ }^{(9)}$.

A amostra foi constituída por 10 integrantes da equipe de enfermagem que presta assistência do pré-natal ao pós-parto, no hospital do estudo. Este representa uma das instituições municipais de referência na prestação de serviços às puérperas na região. $\mathrm{O}$ número de participantes foi definido pelo critério de saturação das falas nas entrevistas.

Os dados foram coletados por meio de entrevistas realizadas no mês de outubro de 2009 e norteadas por questões subjetivas sobre conteúdos teóricos e habilidades relacionadas ao cuidado à paciente e relativas a problemas relacionados com a lactação.

Esses dados foram interpretados mediante análise temática, seguindo os critérios de organização, exaustividade e análise final ${ }^{(10)}$. A análise das entrevistas permitiu categorizar os seguintes temas; problemas maternos relacionados ao aleitamento; medidas adotadas pela equipe de enfermagem para a prevenção dos problemas maternos relacionados ao aleitamento; e responsabilidade da equipe de enfermagem para a prevenção dos problemas maternos relacionados ao aleitamento.

O estudo foi aprovado pelo Comitê de Ética em Pesquisa da Faculdade Integral Diferencial (FACID), sob o parecer n. 186/09. O estudo respeitou a Resolução 196/96 do Conselho Nacional de Saúde sobre pesquisa envolvendo seres humanos ${ }^{(11)}$ e as determinações da Convenção de Helsinkii ${ }^{(12)}$. Os participantes assinaram o termo de consentimento livre e esclarecido e para garantir o seu anonimato, os nomes foram substituídos por nomes de cores. 


\section{RESULTADOS}

\section{Perfil dos sujeitos da pesquisa}

Os profissionais de enfermagem participantes do estudo tinham idade entre 25 a 61 anos, sendo todos do sexo feminino. Quatro entrevistadas eram enfermeiras, três eram técnicas em enfermagem e três auxiliares de enfermagem. O tempo de formação variou de 2 anos e 6 meses a 10 anos para as enfermeiras; de 16 a 27 anos para as técnicas; e entre 16 a 20 anos para as auxiliares de enfermagem. O tempo de atuação na maternidade variou de 3 meses a 6 anos entre as enfermeiras, e de 4 a 21 anos entre técnicas em enfermagem e auxiliares de enfermagem.

\section{Problemas maternos relacionados ao aleitamento}

Entre os problemas maternos relacionados à lactação, a equipe de enfermagem relatou o desmame precoce motivado pela falta de conhecimento materno sobre o aleitamento, o que pode ser identificado nas descrições apresentadas a seguir transcritas:

Desconhecimento da importância da amamentação. (Vermelho)

O maior problema é a questão da conscientização; achar que no primeiro dia o leite é pouco; não acreditar que irá aumentar a quantidade nos dias seguintes. (Roxo)

[...] não conhece a amamentação direito e acha que ficar sem amamentar não pode trazer complicações para o bebê e até pra ela própria. (Preto)

Segundo a experiência relatada, a falta de informação e de conhecimento, por parte de algumas mães, sobre a prática do aleitamento, também contribui para o surgimento de complicações:

Mastite, ingurgitamento mamário e fissuras. (Verde)

Fissuras mamilares, ingurgitamento e mastites com e sem abscesso. (Amarelo)

Dor, fissuras, medo de amamentar pelo fato de sentirem dores, mastite. (Laranja)

Medidas adotadas pela equipe de enfermagem para a prevenção dos problemas maternos relacionados ao aleitamento

Como medidas adotadas diante das complicações da amamentação, as seguintes ações foram referidas:

Compressa fria; hidratar os mamilos com o próprio leite. (Laranja)

São realizados curativos, limpeza correta no mamilo, ordenha e compressa. (Verde)

$O$ correto é orientar a continuar a amamentar $e$, dependendo da situação, suspender a amamentação temporariamente, voltando quando tão logo não ocorrer a presença de pus. (Roxo)

Ao analisar as medidas adotadas para a prevenção dos problemas de lactação, a equipe de enfermagem descreveu atividades como:

Orientação desde a admissão da paciente até após o parto. (Branco)

Orientações, palestras, demonstrações de técnicas de amamentação. (Verde)

Orientações sobre o correto posicionamento do recém-nascido junto ao seio materno, sobre o não-uso de complementos alimentares durante os seis primeiros meses, não-uso de chupetas e mamadeiras e sobre os inúmeros benefícios da amamentação. (Branco)

Pega do bebê; posicionamento ou postura durante a amamentação; ordenha manual; exposição ao sol das mamas. (Azul)

Observou-se, assim, que os membros da equipe percebem a educação em saúde como importante para prevenir problemas na lactação.

\section{Responsabilidade da equipe de enfermagem na prevenção dos problemas maternos relacionados ao aleitamento}

Quanto a esse tema, os profissionais de enfermagem citaram as seguintes ações: 
Tentar prevenir problemas de amamentação com orientações, preparo do mamilo durante a gravidez e incentivo ao aleitamento exclusivo. (Verde)

A Enfermagem trabalha com a prevenção, portanto exerce papel fundamental na prevenção de complicações na amamentação. (Amarelo)

\section{DISCUSSÃO}

$\mathrm{O}$ conhecimento de problemas relacionados à lactação é de grande importância para a equipe de enfermagem, pois as avaliações feitas em puérperas, por meio de anamnese, histórico e exame físico possibilitam a verificação dos fatores de risco associados, de forma a estabelecer os cuidados necessários para minimizar os efeitos adversos ao binômio mãe e filho.

Muitos fatores contribuem para o desmame precoce, e a falta de conhecimento sobre aleitamento materno, por parte das mães, tem papel importante na redução da amamentação. A carência de informações sobre as práticas de amamentar foi relatada; entre as justificativas para o desmame precoce estão dúvidas sobre a qualidade nutricional do leite e o choro do bebêê.

Outro grande problema na amamentação é a descrença da mulher acerca da sua capacidade em produzir leite em quantidade adequada. Assim, informá-la e ajudá-la a perceber os fatores que podem interferir no processo de amamentação é a principal atitude a ser tomada pelos profissionais da saúde ${ }^{(1)}$.

A intervenção educativa tende a tornar-se um fator em potencial para o estímulo ao aleitamento materno. Para isso, o profissional de enfermagem precisa não só ter conhecimentos e habilidades, mas estar suficientemente sensibilizado para incorporá-los em sua prática $^{(6)}$.

Por outro lado, a ocorrência de complicações pode levar a problemas cada vez mais sérios, dificultando os cuidados a serem prestados com vistas à assistência primária adequada. As intercorrências nas mamas durante o puerpério representam importantes fatores de desmame precoce no Brasil ${ }^{(14)}$. A predisposição à mastite inclui mamadas com horários irregulares, redução súbita no número de mamadas, longo período de sono do bebê, uso de chupetas ou mamadeiras, não esvaziamento completo das mamas, produção excessiva de leite, separação entre mãe e bebê, e desmame abrupto $^{(5)}$.

Quanto ao ingurgitamento mamário, que em geral se inicia entre o terceiro e o sétimo dia após o parto, considera-se que sua principal causa pode ser a remoção ineficiente de leite por separação entre mãe e bebê, mamadas muitos espaçadas e técnica inadequada de amamentação, sendo sua prevenção possível mediante técnica adequada de amamentação, mamadas em livre demanda, não-utilização de suplementos e remoção efetiva do leite ${ }^{(15)}$.

No início das mamadas, a maioria das mulheres sente uma discreta dor ou desconforto, o que pode ser considerado normal; no entanto, mamilos muito dolorosos e machucados não são normais, sendo os traumas mamilares por posicionamento e pega inadequados as causas mais comuns de dor ${ }^{(16)}$.

O Ministério da Saúde recomenda que nos casos de ingurgitamento mamário patológico devem ser adotadas medidas como: ordenha manual da aréola; mamadas freqüentes (livre demanda); massagens delicadas nas mamas, particularmente nas regiões mais afetadas pelo ingurgitamento; uso de analgésicos ou anti-inflamatórios, auxiliando na redução da inflamação e do edema; suporte para as mamas, como o uso ininterrupto de sutiã com alças largas e firmes; e, no caso de o bebê não sugar, realizar ordenha da mama manualmente ou com bomba de sucção e aplicar compressas frias ou gelo envolto em tecido ${ }^{(17)}$.

No entanto, é importante destacar que o tempo de aplicação das compressas frias não deve ultrapassar 20 minutos, devido ao efeito rebote, ou seja, um aumento de fluxo sanguíneo para compensar a redução da temperatura local. As compressas frias provocam vasoconstricção temporária pela hipotermia, o que leva à redução do fluxo sanguíneo, com consequente redução do edema, aumento da drenagem linfática e menor produção do leite, devido à redução da oferta de substratos necessários à produção do leite ${ }^{(17)}$.

Dessa forma, uma boa atuação no sentido de promover, proteger e apoiar a amamentação requer, além de conhecimentos sobre aleitamento materno, também habilidades clínicas e de aconselhamento ${ }^{(16)}$. As atenções e orientações voltadas às mulheres sobre o percurso na amamentação, objetiva a prevenção e devem ser iniciadas na gestação, desde o período do pré-natal até o puerpério.

É preciso que os profissionais da área da saúde, em particular os enfermeiros, percebam a importância da comunicação como instrumento do processo de trabalho em saúde, descobrindo novas opções, rupturas e continuidades em seu compromisso social ${ }^{(18)}$. As ações do indivíduo dependerão de sua interação 
com os outros sujeitos e consigo mesmo, e de sua cultura, devendo o enfermeiro agir como facilitador, ao fornecer informação e cuidado ${ }^{(19)}$.

O profissional de saúde deve identificar, durante o pré-natal, os conhecimentos, a experiência prática, as crenças, e a vivência social e familiar da gestante, a fim de promover educação em saúde para o aleitamento materno, assim como garantir vigilância e efetividade durante a assistência à nutriz no pós-parto ${ }^{(20)}$.

Compreendendo a amamentação como um processo complexo que envolve a cultura, o valor, o social, o biológico e o emocional, deve-se ir além das orientações quanto ao manejo, sendo fundamental que o enfermeiro cuide não somente com abordagem técnica, mas que essa seja ampliada por meio da assistência associada aos aspectos socioculturais da amamentação ${ }^{(19)}$.

A equipe de enfermagem tem a responsabilidade de apoiar as mulheres por meio de atitudes, que podem influenciar positivamente o início da amamentação, evitar o desmame precoce e diminuir o surgimento de possíveis complicações. Com a implantação do Programa Nacional de Incentivo ao Aleitamento Materno, iniciou-se um processo de conscientização dos profissionais ao enfatizar a responsabilidade de todos na promoção, incentivo e apoio a essa prática ${ }^{(20)}$.

\section{CONCLUSÃO}

As categorias que surgiram nas falas dos entrevistados demonstram a visão da equipe de enfermagem quanto aos problemas relacionados à amamentação, observando-se que esses profissionais entendem que o processo de aleitamento materno deve ser iniciado precocemente, e que as puérperas têm dúvidas e dificuldades relativas à amamentação.

A equipe de enfermagem possui um papel fundamental no acompanhamento de puérperas em ações de promoção à saúde, assistência e prestação de serviços de qualidade no preparo de mulheres para o aleitamento materno e nas ações de educação em saúde, com o intuito de melhorar o enfrentamento dos problemas relacionados à amamentação. O acompanhamento da mulher pelas equipes de Saúde da Família, desde o pré-natal ao puerpério imediato e pós-parto, pode contribuir para dirimir dúvidas e promover a amamentação.

\section{REFERÊNCIAS}

1. Carvalho CM, Bica OSC, Moura GMSS. Consultoria em aleitamento materno no hospital de clínicas de Porto Alegre. Rev HCPA. 2007;27(2):53-6.

2. Ministério da Saúde (BR). Secretaria de Políticas de Saúde. Área de Saúde da Criança. Prevalência do aleitamento materno nas capitais brasileiras e no Distrito Federal. Brasília: Ministério da Saúde; 2001.

3. Giugliani ERJ. Falta embasamento científico no tratamento dos traumas mamilares. J Pediatr. 2003;79(3):197-8.

4. Lamounier JÁ, Moulin ZS, Xavier CC. Recomendações quanto à amamentação na vigência de infecção materna. J Pediatr. 2004;80(5):181-8.

5. Barros SMO, Marin HF, Abrão ACFV. Enfermagem obstétrica e ginecológica: guia para a prática assistencial. São Paulo: Roca; 2002.

6. Moreira PL, Fabbro MRC. Utilizando técnicas de ensino participativas como instrumento de aprendizagem e sensibilização do manejo da lactação para profissionais de enfermagem de uma maternidade. Acta Paul Enferm. 2005;18(3):320-5.

7. Driessnack M, Sousa VD, Mendes IAC. Revisão dos desenhos de pesquisa relevantes para enfermagem: parte 2: desenhos de pesquisa qualitativa. Rev LatinoAm Enfermagem. 2007;15(4):684-8.

8. Wood GL, Haber J. Pesquisa em enfermagem: métodos, avaliação crítica e utilização. Rio de Janeiro: Guanabara Koogan; 2001.

9. Oliveira SL. Tratado de metodologia científica: projetos de pesquisa, TGI, TCC, monografia, dissertações e teses. $2^{\text {a }}$ ed. São Paulo: Pioneira; 2000.

10. Minayo MCS. O desafio do conhecimento: pesquisa qualitativa em saúde. $7^{\mathrm{a}}$ ed. São Paulo: Hucitec/ Abrasco; 2008.

11. Ministério da Saúde (BR). Conselho Nacional de Saúde. Diretrizes e normas regulamentadores de pesquisa envolvendo seres humanos. Resolução n. 196, de 10 de oututbo de 1996. Brasília; 1996.

12. World Medical Association. Declaration of Helsinki. Ethical principles for medical research involving human subjects. 59th WMA General Assembly, Seoul October; 2008. 
13. Percegoni N, Araujo RMA, Silva MMS, Euclydes MP, Tinôco ALA. Conhecimento sobre aleitamento materno de puérperas atendidas em dois hospitais de Viçosa, Minas Gerais. Rev Nutr. 2002;15(1):29-35.

14. Sales CM, Seixas SC. Causas de desmame precoce no Brasil. Cogitare Enferm. 2008;13(3):443-7.

15. Lopez FA, Campos Junior D. Tratado de pediatria. Sociedade Brasileira de Pediatria. Barueri: Manole; 2007.

16. Giugliani ERJ. O aleitamento materno na prática clínica. J. Pediatr. 2000;76(3):238-52.

17. Ministério da Saúde (BR). Secretaria de Atenção à Saúde. Departamento de Atenção Básica. Saúde da criança: nutrição infantil. Aleitamento materno e alimentação complementar. Série A. Normas e manuais técnicos. Cadernos de atenção básica, n.23. Brasília: Ministério da Saúde; 2009.

18. Machado LV, Larocca LM. Intercorrências mamárias e desmame precoce - uma abordagem comunicacional. Cogitare Enferm. 2004;9(2):89-98.

19. Almeida IS, Ribeiro IB, Rodrigues BMRD, Costa CCP, Freitas NS, Vargas EB. Amamentação para mães primíparas: perspectivas e intencionalidades do enfermeiro ao orientar. Cogitare Enferm. 2010;15(1):1925.

20. Almeida NAM, Fernandes A.G, Araújo CG. Aleitamento materno: uma abordagem sobre o papel do enfermeiro no pós-parto. Rev Eletr Enf. 2004;6(3):35867. 